\title{
Level V Lateral Neck Dissection in FNA-proven Papillary Thyroid Carcinoma
}

\author{
Mohamad S Farahat ${ }^{1}$, Mohamed K Kamel ${ }^{2}$, Doaa A Saad ${ }^{3}$, Hosam M Hamza ${ }^{4}$
}

\begin{abstract}
Aim and objective: Papillary thyroid carcinoma (PTC) constitutes $80 \%$ of thyroid cancer cases. It has a high risk for lymph node metastases in both central and lateral compartments of the neck. This study is an attempt to better define the need for level V dissection in PTC, possible predictors of involvement, and rate of complications.

Materials and methods: In the General Surgery Department, Minia University Hospital, Egypt, 35 patients diagnosed with PTC were subjected to lateral neck dissection (LND) in the period from October 2015 till January 2019. In LND, we spared the internal jugular vein, spinal accessory nerve, and sternocleidomastoid muscle. All neck specimens were intraoperatively labeled by level for the pathologist.

Results: Ten patients (28.6\%) were males and 25 (71.4\%) were females. Total level V metastases were detected in 20 specimens (38.5\%) all having level Vb metastases except three specimens having metastases in level Va (no skip metastases). Level III metastases were more frequent than level $\mathrm{V}$ and level II. $67.3 \%$ of specimens were positive for extrathyroidal extension (ETE) and $57.7 \%$ positive for lymphovascular invasion. Injury of the spinal accessory nerve was detected in one case; $1.9 \%$ and chylous leak in one case; $1.9 \%$.

Conclusion: In experienced hands, including level V leads to better local control without a high rate of complications. We concluded that formal modified radical neck dissection (MRND) is necessary to reduce the morbidity of reoperation surgery in PTC.

Keywords: Chylous leak, Lateral neck dissection, Level V metastases, Lymph nodes, Papillary thyroid carcinoma, Spinal accessory nerve.

World Journal of Endocrine Surgery (2021): 10.5005/jp-journals-10002-1315
\end{abstract}

\section{INTRODUCTION}

Well-differentiated thyroid carcinoma (WDTC) is the most common endocrine malignancy regardless of ethnicity or of geographic location. ${ }^{1}$ Papillary thyroid carcinoma (PTC) constitutes approximately $80 \%$ of all thyroid cancer cases ${ }^{2}$ and the incidence is on the rise. ${ }^{3}$

In general, WDTC has a high and well-known propensity to spread to lymph nodes in the neck, in both the central and lateral compartments. ${ }^{4}$

Although the pattern of lymphatic spread is well known, the impact of nodal metastases on survival is still a controversial issue, ${ }^{5}$ even though current staging systems consider nodal metastases to confer a poorer prognosis, particularly in older patients. ${ }^{6}$

Relatively good prognosis of patients with WDTC, the unclear prognostic value of nodal disease, ${ }^{7}$ and possible complications related to neck dissection (neural damage, shoulder syndrome, chylous leak, and hemorrhage), ,,8 are all factors contributing to the lack of clear indications for management of node-positive patients.

The neck levels to be removed in neck dissection for WDTC is one of the most debated issues; particular emphasis has been placed on levels I, V, and sublevel IIb. ${ }^{9}$

This prospective study explores the debate about level $\mathrm{V}$ lateral neck dissection (LND) in fine-needle aspiration (FNA)-proven PTC. Our work is an attempt to better define the need for level V dissection in PTC, analyzing the distribution of metastatic nodes in each subdivision, possible predictors of involvement, and rate of complications.

\footnotetext{
${ }^{1-4}$ Department of General Surgery, Faculty of Medicine, Minia University, Egypt
}

Corresponding Author: Hosam M Hamza, Department of General Surgery, Faculty of Medicine, Minia University, Egypt, e-mail: hossam. hamza@mu.edu.eg

How to cite this article: Farahat MS, Kamel MK, Saad DA, et al. Level V Lateral Neck Dissection in FNA-proven Papillary Thyroid Carcinoma. World J Endoc Surg 2021;13(1):4-8.

Source of support: Nil

Conflict of interest: None

\section{Patients and Methods}

\section{Patients}

A total number of 35 patients with PTC subjected to LND surgery (levels II, III, IV, Va, and Vb) were enrolled in this study. Surgery was done in the General Surgery Department, Minia University Hospital, Egypt in the period from October 2015 until January 2019 after approval of the institutional board of ethics. Unilateral neck dissection was done in 18 patients. Seventeen patients underwent bilateral dissection (each neck side is considered as one specimen) so the total number of specimens is 52 . Fully informed consent was taken from the patients before commencement.

The following inclusion and exclusion criteria were enrolled. The inclusion criteria were: pathologically proven PTC, evidence of

() The Author(s). 2021 Open Access This article is distributed under the terms of the Creative Commons Attribution 4.0 International License (https://creativecommons. org/licenses/by-nc/4.0/), which permits unrestricted use, distribution, and non-commercial reproduction in any medium, provided you give appropriate credit to the original author(s) and the source, provide a link to the Creative Commons license, and indicate if changes were made. The Creative Commons Public Domain Dedication waiver (http://creativecommons.org/publicdomain/zero/1.0/) applies to the data made available in this article, unless otherwise stated. 
lateral lymph node metastasis by histopathological examination, primary cases needing simultaneous total thyroidectomy and neck dissection, and cases presenting with lateral neck disease after the previous thyroidectomy. Cases with less than a selective neck dissection of levels II through $\mathrm{V}$, an incomplete pathology report, pathologic features other than PTC, distant metastasis, or a history of LND were excluded from this study. Central neck tissue (level VI) was not included in our study.

\section{Methods}

Preoperative physical examination, high-quality thyroid ultrasonography (US), and US-guided FNA of the primary tumor and the suspicious lymph nodes were done to all patients.

In primary cases, total thyroidectomy is done first, followed by LND sparing the internal jugular vein, spinal accessory nerve, and sternocleidomastoid muscle (Fig. 1). All neck specimens were intraoperatively labeled by level for the pathologist.

The neck levels were defined by the following boundaries: level II: from the base of the skull to the inferior border of the hyoid bone; level III: between the hyoid and the inferior border of the cricoid cartilage; level IV: between the inferior border of the cricoid cartilage and the clavicle; level V: from the convergence of the sternocleidomastoid and trapezius muscles down to the clavicle.

Demographic and operative data of all patients included age (whether less or more than 55 years), sex, whether neck dissection with total thyroidectomy or previous thyroidectomy, unilateral or bilateral dissection.

Pathological data recorded (in the 52 specimens) include pathological tumor type, tumor diameter (less or more than $1 \mathrm{~cm}$ ), extrathyroidal extension (ETE) including gross and microscopic extension, lymphovascular invasion, and incidence of lymph node metastases in level II, III, IV and level V, Va, Vb for each side (a bilateral case is considered as two specimens). Incidence of surgical complications common in level V dissection was recorded including chylous leak and spinal accessory nerve injury presenting as "shoulder syndrome" in the form of pain, limited abduction of the shoulder, full passive range of motion, and anatomical deformities such as scapular flaring, droop, and protraction.

We classified the age as above or below 55 years as this age is widely used as a clinical marker for prognosis. Also, the tumor size less or more than $1 \mathrm{~cm}$ as the global definition of microcarcinoma is above that size.

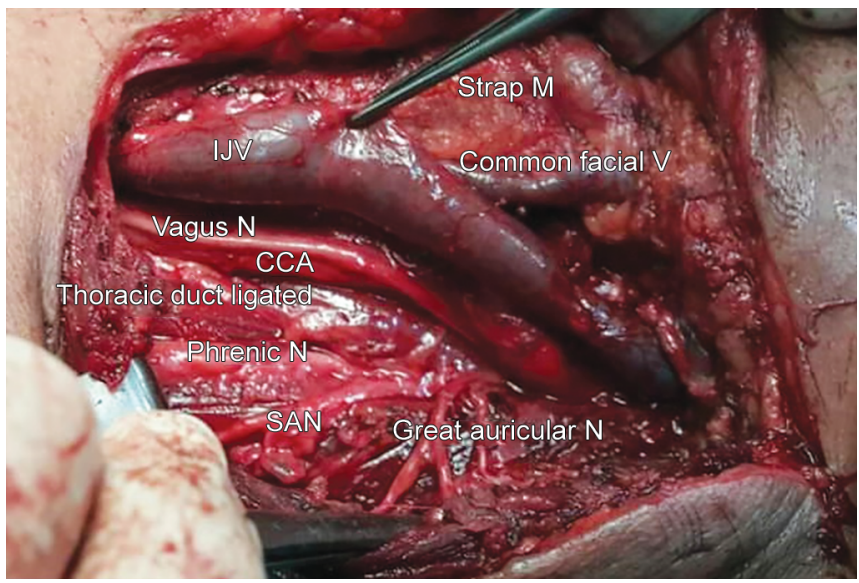

Fig. 1: Modified radical neck dissection including level $V$ sparing the internal jugular vein (IJV) and spinal accessory nerve (SAN) (strap M, strap muscles; CCA, common carotid artery; N, nerve)

\section{Statistical Analysis}

The collected data were coded, tabulated, and statistically analyzed using SPSS program (Statistical Package for Social Sciences) software version 25. Descriptive statistics were done for categorical data by number and percentage. Analyses were done for qualitative data using the Chi-square test (if $<20 \%$ of cells have expected number per cell $<5$ ) or Fisher's exact test (if $>20 \%$ of cells have expected number per cell $<5$ ). Simple logistic regression analysis for prediction of total level $\mathrm{V}$ metastases. The level of significance was taken at $p$ value $<0.05$.

\section{Results}

Of the patients enrolled in our study $(n=35), 10$ (28.6\%) were males and 25 (71.4\%) were females. Demographic data for our patients is presented in Table 1.

Regarding the characteristics of specimens (presented in Table 2), PTC was shown in $80 \%$ of specimens, follicular variant in $11.4 \%$, and other types of papillary carcinoma in $8.6 \%$.

Total level V metastases were detected in 20 specimens (38.5\%) all having level $\mathrm{Vb}$ metastases except three specimens having metastases in level Va (no skip metastases). Level III metastases were more frequent than level V and level II (80.8\%, 67.3, 51.9\%, respectively). Microcarcinoma was present only in $7.7 \%$ of specimens. $67.3 \%$ of specimens were positive for ETE, and $57.7 \%$ positive for lymphovascular invasion.

Postoperative complications during level $\mathrm{V}$ dissection (presented in Table 3) included injury of the spinal accessory nerve (one case; $1.9 \%$ ) and chylous leak (one case; $1.9 \%$ ).

During our study, no significant relation was detected between patient characteristics (including age, sex, and cell pathology) and total level $\mathrm{V}$ metastases (Table 4).

Table 4 also demonstrates the relation between total level $V$ metastases and the specimen characteristics; including tumor diameter (less or more than $1 \mathrm{~cm}$ ), ETE, lymphovascular invasion, the incidence of lymph node metastases in levels II, III, and IV. A significant relation was demonstrated between the lymphovascular invasion, level II metastases, and total level V metastases ( $p$ value 0.046 and $<0.001$, respectively).

Table 1: Patients' characteristics

\begin{tabular}{ll}
\hline Characteristic & Total $(n=35)(\%)$ \\
\hline Age (years) & $13(37.1)$ \\
$>45$ & $22(62.9)$ \\
$<45$ & \\
Sex & $10(28.6)$ \\
Male & $25(71.4)$ \\
Female & \\
ND & $19(54.3)$ \\
Primary treatment & $16(45.7)$ \\
Previous treatment & \\
Side & $18(51.4)$ \\
Unilateral & $17(48.6)$ \\
Bilateral & \\
Cell pathology & \\
Papillary & $28(80)$ \\
Follicular variant & $4(11.4)$ \\
Others & $3(8.6)$ \\
\hline
\end{tabular}


Table 2: Specimens' characteristics (mets; metastases)

\begin{tabular}{lc}
\hline Characteristic & Total $(n=52)(\%)$ \\
\hline Total level V mets & $32(61.5)$ \\
Negative & $20(38.5)$ \\
Positive & \\
Level Va mets & $49(94.2)$ \\
Negative & $3(5.8)$ \\
Positive & \\
Level Vb mets & $32(61.5)$ \\
Negative & $20(38.5)$ \\
Positive & \\
Tumor diameter & $4(7.7)$ \\
$<1$ cm & $48(92.3)$ \\
$>1$ cm & \\
Extracapsular spread & $17(32.7)$ \\
Negative & $35(67.3)$ \\
Positive & \\
Lymphovascular invasion & \\
Negative & $22(42.3)$ \\
Positive & $30(57.7)$ \\
Level II mets & \\
Negative & $25(48.1)$ \\
Positive & $27(51.9)$ \\
Level III mets & \\
Negative & $10(19.2)$ \\
Positive & $42(80.8)$ \\
Level IV mets & \\
Negative & \\
Positive & \\
\end{tabular}

Table 3: Postoperative complications

\begin{tabular}{lc}
\hline Spinal accessory nerve injury & \\
Negative & $51(98.1)$ \\
Positive & $1(1.9)$ \\
Chylous leak & \\
Negative & $51(98.1)$ \\
Positive & $1(1.9)$ \\
\hline
\end{tabular}

By using simple logistic regression analysis for prediction of total level V metastases, level II metastases represent the only variable showing significant association with level $\mathrm{V}$ metastasis $(p=0.003$, Table 5). Odds ratio 14.7, confidence interval 2.5-86.5.

\section{Discussion}

In patients with PTC, the incidence of regional lymph node metastases (LNM) is quite common, approximately $50-80 \%$ based on previous reports. Although PTC patients generally have a fine prognosis, omitting level $V$ during LND potentially misses level $V$ disease in one-fifth of patients. ${ }^{10}$ The 2015 American Thyroid Association (ATA) guidelines recommend therapeutic LND (encompassing level II to V) for patients with biopsy-proven metastatic lateral cervical lymphadenopathy (N1b). ${ }^{11}$

In addition, some authors supposed that it was difficult to distinguish metastatic lymph nodes in level $\mathrm{V}$ by physical
Table 4: Relation between patient characteristics and total level V metastases and the relation between specimen characteristics and total level $\mathrm{V}$ metastases (Chi-square test "if expected values within cell $\geq 5$ " and Fisher's exact test "if expected values within cell $<5$ " for qualitative data between the two groups)

\begin{tabular}{|c|c|c|c|}
\hline & \multicolumn{2}{|c|}{ Total level $\mathrm{V}$ mets } & \multirow[b]{3}{*}{$p$ value } \\
\hline & Negative & Positive & \\
\hline & $N=32(\%)$ & $N=20(\%)$ & \\
\hline \multicolumn{4}{|l|}{ Age (years) } \\
\hline$>45$ & $10(31.3)$ & $10(50)$ & 0.176 \\
\hline$<45$ & $22(68.8)$ & $10(50)$ & \\
\hline \multicolumn{4}{|l|}{ Sex } \\
\hline Male & $10(31.3)$ & $5(25)$ & 0.628 \\
\hline Female & $22(68.8)$ & $15(75)$ & \\
\hline \multicolumn{4}{|l|}{ Cell pathology } \\
\hline Papillary & $24(75)$ & $17(85)$ & 0.777 \\
\hline Follicular variant & $4(12.5)$ & $2(10)$ & \\
\hline Others & $4(12.5)$ & $1(5)$ & \\
\hline \multicolumn{4}{|l|}{ Tumor diameter } \\
\hline$<1 \mathrm{~cm}$ & $2(6.3)$ & $2(10)$ & 0.634 \\
\hline$>1 \mathrm{~cm}$ & $30(93.8)$ & $18(90)$ & \\
\hline \multicolumn{4}{|c|}{ Extrathyroidal extension } \\
\hline Negative & $11(34.4)$ & $6(30)$ & 0.744 \\
\hline Positive & $21(65.6)$ & $14(70)$ & \\
\hline \multicolumn{4}{|c|}{ Lymphovascular invasion } \\
\hline Negative & $17(53.1)$ & $5(25)$ & $0.046^{*}$ \\
\hline Positive & $15(46.9)$ & $15(75)$ & \\
\hline \multicolumn{4}{|l|}{ Level II mets } \\
\hline Negative & $22(68.8)$ & $3(15)$ & $<0.001^{*}$ \\
\hline Positive & $10(31.3)$ & $17(85)$ & \\
\hline \multicolumn{4}{|l|}{ Level III mets } \\
\hline Negative & $5(15.6)$ & $5(25)$ & 0.404 \\
\hline Positive & $27(84.4)$ & $15(75)$ & \\
\hline \multicolumn{4}{|l|}{ Level IV mets } \\
\hline Negative & $10(31.3)$ & $7(35)$ & 0.779 \\
\hline Positive & $22(68.8)$ & $13(65)$ & \\
\hline
\end{tabular}

*Significant level at $p$ value $<0.05$

Table 5: Simple logistic regression analysis for prediction of total leve $\mathrm{V}$ mets (OR: odds ratio; $\mathrm{Cl}$ : confidence interval)

\begin{tabular}{llll}
\hline & OR & $95 \% \mathrm{Cl}$ & pvalue \\
\hline $\begin{array}{l}\text { Lymphovascular } \\
\text { invasion }\end{array}$ & 3.3 & $0.7-13.9$ & 0.112 \\
Level II mets & 14.7 & $2.5-86.5$ & $0.003^{*}$ \\
\hline
\end{tabular}

*Significant level at $p$ value $<0.05$

examination, US, and even CT scan and that it might lead to local recurrence by LND. ${ }^{12,13}$ Therefore, the authors emphasized that comprehensive LND including level II-V was necessary and vital for N1b patients with PTC.

However, some recent studies have opposed extensive LND since it has the potential for increased morbidity, such as spinal accessory nerve injury, and no significant benefit of survival compared with selective neck dissection (SND). ${ }^{14}$ A study by 
Xu et al. ${ }^{15}$ showed that the 5 -year ipsilateral regional recurrence rate was $8 \%$ and $9 \%$ with selective and comprehensive dissection, respectively ( $p=0.89$ ), and concluded that the extent of neck dissection did not predict the probability of regional recurrence in PTC patients presenting with lateral neck metastases. Thus, the dilemma regarding the proper extent of LND, especially whether level V should be routinely dissected, remains to be solved.

Our study is in an attempt to better define the need for level $V$ dissection in FNA-proven PTC. We investigated the incidence of level $\mathrm{V}$ metastases, the rate of common postoperative complications associated with level $\mathrm{V}$ dissection, and the predictive factors for level $\mathrm{V}$ metastases to detect which patient deserves the risk of this surgery.

In our study, the rate of total level $\mathrm{V}$ metastases was significant (38.5\%) and this ranged from 8 to $25.3 \%$ in other series. 5,16

All cases with level $\mathrm{V}$ metastases had level $\mathrm{Vb}$ metastases except three specimens having metastases also in level Va (5.8\%) that means there were no skip metastases. This is consistent with a meta-analysis by Eskander and co-workers ${ }^{17}$ that reported level V metastatic disease in $25.3 \%$ of cases, with Va and Vb nodal positivity in 7.9 and $21.5 \%$ of patients, respectively. This evidence leads them to recommend a comprehensive selective neck dissection of levels Ila, Ilb, III, IV, and Vb in patients with lateral neck disease from PTC.

The evidence for level $\mathrm{Va}$ is lacking, as most studies did not distinguish between levels $\mathrm{Va}$ and $\mathrm{Vb}$, and the border between the two levels was inconsistent. Future studies will need to address these sublevels separately. ${ }^{17}$

The overall rates of metastases in Level III were more frequent than level IV and level II (80.8\%, 67.3, and 51.9\%, respectively). Such high rates of metastases for these three compartments (levels II, III, and IV) were more frequent than level $\mathrm{V}$ which is in line with a previous study by Yanir and Doweck. ${ }^{18}$ Therefore, comprehensive dissection of levels II-IV is necessary for N1b PTC patients.

Since level $\mathrm{V}$ metastases are vital for prognosis but their dissection may have an unfavorable impact on shoulder function and other aspects, and also the sensitivity of preoperative imaging is particularly poor for detecting the presence of these metastases, ${ }^{19}$ therefore, we investigated the predictive factors of level $\mathrm{V}$ metastasis to help establish indications for level $\mathrm{V}$ dissection in clinically suspicious N1b PTC patients. During our study, a significant relation was demonstrated between the lymphovascular invasion, level II metastases, and total level V metastases and by using simple logistic regression analysis for prediction of total level $V$ metastases, level II metastases represent the only variable showing significant association with level $\mathrm{V}$ metastasis. Other studies have also shown that simultaneous multi-level metastases are an independent predictor for level V metastasis. ${ }^{20,21}$

Significant shoulder disability after comprehensive neck dissection including levels Ilb and V occurs in up to $40 \%$ of patients, although much of this may be temporary..$^{15}$ In our study, we had one case $(1.9 \%)$ of spinal accessory nerve injury during level $\mathrm{V}$ dissection. A chylous leak in the neck is a very rare complication after a thyroidectomy. The risk of this complication increases when the thyroid surgery is associated with neck dissection $(5.1 \%$ after total thyroidectomy and LND on one side, 6.2\% after total thyroidectomy, and LND on both sides). ${ }^{16}$ Only one of our cases (1.9\%) had a chylous leak.

\section{LIMITATIONS}

Our study had some limitations in the term of sample size. We presented a relatively small number of patients and further clinical studies in the same topic with a larger population are still needed.

\section{Conclusion}

This study indicates that level $\mathrm{V}$ metastases in patients with PTC are significant especially in level $\mathrm{Vb}$. The desire to avoid level $\mathrm{V}$ dissection can put those patients at increased risk for persistent and recurrent disease, which adds to long-term morbidity. In experienced hands, including level V will lead to better local control without conferring a high rate of complications, and should be assessed on an individual basis according to the predictive factors. We conclude that formal modified radical neck dissection (MRND) is necessary to reduce the morbidity of reoperation surgery, we suggest it be adopted more uniformly in the management of LND in patients with PTC.

\section{References}

1. Kim W. A closer look at papillary thyroid carcinoma. Endocrinol Metab (Seoul) 2015;30(1):1-6. DOI: 10.3803/EnM.2015.30.1.1.

2. Zhu X, Yao J, Tian W. Microarray technology to investigate genes associated with papillary thyroid carcinoma. Mol Med Rep 2015;11(5):3729-3733. DOI: 10.3892/mmr.2015.3180.

3. Raposo L, Morais S, Oliveira MJ, et al. Trends in thyroid cancer incidence and mortality in Portugal. Eur J Cancer Prev 2017;26(2):135143. DOI: $10.1097 /$ CEJ.0000000000000229.

4. Dralle $H$, Machens A. Surgical management of the lateral neck compartment for metastatic thyroid cancer. Curr Opin Oncol 2013;25(1):20-26. DOI: 10.1097/CCO.0b013e328359ff1f.

5. Stack BC Jr, Ferris RL, Goldenberg D, et al. American Thyroid Association consensus review and statement regarding the anatomy, terminology, and rationale for lateral neck dissection in differentiated thyroid cancer. Thyroid 2012;22(5):501-508. DOI: 10.1089/thy.2011.0312.

6. Wang L, Ganly I. Nodal metastases in thyroid cancer: prognostic implications and management. Future Oncol 2016;12(7):981-994. DOI: $10.2217 /$ fon.16.10.

7. Hay ID. Papillary thyroid carcinoma. Endocrinol Metab Clin North Am 1990;19(3):545-576. DOI: 10.1016/S0889-8529(18)30310-4.

8. Madenci AL, Caragacianu D, Boeckmann J, et al. Lateral neck dissection for well-differentiated thyroid carcinoma: a systematic review. Laryngoscope 2014;124(7):1724-1734. DOI: 10.1002/ lary.24583.

9. Wang $Y$, Guan $Q$, Xiang J. Nomogram for predicting level V lymph node metastases in papillary thyroid carcinoma with clinically lateral lymph node metastases: a large retrospective cohort study of 1037 patients from FDUSCC. J Cancer 2019;10(3):772. DOI: 10.7150/ jca.28527.

10. Javid M, Graham E, Malinowski J, et al. Dissection of levels II through V is required for optimal outcomes in patients with lateral neck lymph node metastasis from papillary thyroid carcinoma. J Am Coll Surg 2016;222(6):1066-1073. DOI: 10.1016/j.jamcollsurg.2016.02.006.

11. Haugen BR, Alexander EK, Bible KC, et al. 2015 American Thyroid Association management guidelines for adult patients with thyroid nodules and differentiated thyroid cancer. Thyroid 2015;26(1):1. DOI: 10.1089/thy.2015.0020.

12. Shim MJ, Roh JL, Gong G, et al. Preoperative detection and predictors of level $\mathrm{V}$ lymph node metastasis in patients with papillary thyroid 
carcinoma. Br J Surg 2013;100(4):497-503. DOI: 10.1002/bjs. 9024.

13. Kim SK, Park I, Hur N, et al. Patterns, predictive factors and prognostic impact of multilevel metastasis in N1b papillary thyroid carcinoma. Br J Surg 2017;104(7):857-867. DOI: 10.1002/bjs.10514.

14. Kim SK, Park I, Hur N, et al. Should level V be routinely dissected in N1b papillary thyroid carcinoma? Thyroid 2017;27(2):253-260. DOI: 10.1089/thy.2016.0364.

15. Xu JJ, Yu E, McMullen C, et al. Patterns of regional recurrence in papillary thyroid cancer patients with lateral neck metastases undergoing neck dissection. J Otolaryngol Head Neck Surg 2017;46(1):43. DOI: 10.1186/s40463-017-0221-3.

16. Lundgren $\mathrm{Cl}$, Hall P, Dickman PW, et al. Clinically significant prognostic factors for differentiated thyroid carcinoma: a population-based, nested case-control study. Cancer 2006;106(3):524-531. DOI: 10.1002/ cncr.21653.
17. Eskander A, Merdad M, Freeman JL, et al. Pattern of spread to the lateral neck in metastatic well-differentiated thyroid cancer: a systematic review and meta-analysis. Thyroid 2013;23(5):583-592. DOI: 10.1089/thy.2012.0493.

18. Yanir Y, Doweck I. Regional metastases in well-differentiated thyroid carcinoma: pattern of spread. Laryngoscope 2008;118(3):433-436. DOI: 10.1097/MLG.0b013e31815ae3e4.

19. Kupferman ME, Weinstock YE, Santillan AA, et al. Predictors of level $\mathrm{V}$ metastasis in well-differentiated thyroid cancer. Head Neck 2008;30(11):1469-1474. DOI: 10.1002/hed.20904.

20. Lim YC, Choi EC, Yoon YH, et al. Occult lymph node metastases in neck level V in papillary thyroid carcinoma. Surgery 2010;147(2):241-245. DOI: 10.1016/j.surg.2009.09.002.

21. Zhang XJ, Liu D, Xu DB, et al. Should level V be included in lateral neck dissection in treating papillary thyroid carcinoma? World J Surg Oncol 2013;11(1):304. DOI: 10.1186/1477-7819-11-304. 